\title{
Every member of the core is as respectful as any other
}

\author{
Yasemin Dede $^{1}$ - Semih Koray ${ }^{1}$
}

Received: 27 September 2017 / Accepted: 14 May 2018 / Published online: 5 June 2018

(C) Springer-Verlag GmbH Germany, part of Springer Nature 2018

\begin{abstract}
We strategically separate different core outcomes. The natural counterparts of a core allocation in a strategic environment are the $\alpha$-core, the $\beta$-core and the strong equilibrium, modified by assuming that utility is transferable in a strategic context as well. Given a core allocation $\omega$ of a convex transferable utility (TU) game $v$, we associate a strategic coalition formation game with $(v, \omega)$ in which $\omega$ survives, while most other core allocations are eliminated. If the TU game is strictly convex, the core allocations respected by the TU- $\alpha$-core, the TU- $\beta$-core and the TU-strong equilibrium shrink to $\omega$ only in the canonical family of coalition formation games associated with $(v, \omega)$. A mechanism, which strategically separates core outcomes from noncore outcomes for each convex TU game according to the TU-strong equilibrium notion is reported.
\end{abstract}

Keywords TU game $\cdot$ Core $\cdot$ TU- $\alpha$-core $\cdot$ TU- $\beta$-core $\cdot$ TU-strong equilibrium

JEL Classifications $1.001 \cdot 3.007 \cdot 4.005$

\section{Introduction}

The core is indeed a major stability notion in cooperative game theory. When utility is transferable, one only needs to know the maximal total value of utility that each coalition can secure for itself in order to compute the core allocations. This is the reason why transferable utility (TU) games are deemed to be appropriate to model cooperative

Semih Koray

ksemih@bilkent.edu.tr

1 Department of Economics, Bilkent University, Ankara, Turkey 
interaction. TU games are the simplest objects that contain all the information to tell precisely what allocations the core consists of.

On the other hand, the notion of a TU game is too simple to allow an explanation for the instability of noncore outcomes in terms of individual strategic behaviors. A TU game does not even give a least clue as to how coalitions get formed, how their members coordinate to reach a joint behavior or how they share the total value of their coalition among themselves. There is a rather rich literature aiming to fill in this gap by establishing noncooperative foundations for cooperative solution concepts. This literature exemplified by Kalai et al. (1979), Perez-Castrillo (1992), Lagunoff (1994), Perry and Reny (1994) and Serrano (1995) focuses on providing a strategic environment, preferably reflecting the scenario underlying the core concept, which discerns core outcomes from noncore outcomes. This approach amounts to implementing the core by a noncooperative equilibrium notion.

Another problem associated with the core and dealt with by many researchers is based on the fact that the core of a TU game is rarely a singleton set. In case the core of a TU game is empty, one may try to weaken the stability criteria in an attempt to reload the theory with a "predictive power" if stability is regarded as the major force that drives the outcomes. The multiplicity of core allocations is usually considered as diminishing the "predictive power" of the theory as well. Thus, one is inclined to single out certain core allocations as being "more respectful than others", mainly based upon "additional and normative" criteria from outside the rationale of the core itself. This now gives rise to a natural question concerning what loss our stability analysis incurs, when we reject an abundance of core allocations for the sake of certain "fairness" or similar "normative" criteria.

What we do in this paper is to associate a family of strategic environments with each TU game, which is, however, not meant to discern core outcomes from noncore outcomes this time, but to strategically make a distinction between different core outcomes themselves. In our context, each strategic environment renders certain core allocations meaningful while rejecting others, but all that solely based on the rationale of the core itself, without any reference to any normative criteria. As we are interested in TU games with a nonempty core, we confine ourselves to convex TU games.

For each convex TU game, there are an infinite variety of strategic form games that induce it via the maxmin or the minmax operator, while each strategic form game induces a unique TU game under any of these two operators. We thus obtain a manyto-one matching between strategic form games and convex TU games. It is, in fact, this many-to-oneness that we exploit to distinguish between different core allocations of a convex TU game with a nonsingleton core.

Our approach here resembles in spirit what Selten (1975) does in singling out certain Nash equilibria of a strategic form game $g$ as subgame perfect equilibria of an extensive form game inducing $g$. Starting with a convex TU game $v$, we "go back" to a richer structure - a strategic form game-that induces $v$. The natural counterparts of the core in a strategic framework are the $\alpha$-core, the $\beta$-core and the strong Nash equilibrium introduced by Aumann (1961). When we consider the many-to-one matching yielded by the maxmin operator, it seems natural to employ the $\alpha$-core in the strategic 
background, while the $\beta$-core becomes the appropriate candidate under the minmax operator.

The employment of the maxmin as well as the minmax operator in inducing a TU game from a strategic form game assumes the transferability of utilities introduced as part of the strategic context. Although strategic form games have so far been almost always accompanied by nontransferability of utility, the sheer existence of a strategic structure does not seem to form a natural barrier against transferability of utility as such. We thus modify the notions of $\alpha$-core, $\beta$-core and strong Nash equilibrium by allowing transferability of utility. It is the notions of thereby obtained TU- $\alpha$-core, TU- $\beta$-core and TU-strong equilibrium that we employ in the strategic form games.

Given a convex TU game $v$ and an allocation $\omega$ in the core of $v$, we introduce a strategic form coalition formation game $g$ that induces $v$ via the maxmin as well as the minmax operator in such a way that the strategic counterparts of $\omega$ not only turn out to belong the TU- $\alpha$ and TU- $\beta$-cores, but they also form a TU-strong equilibrium of $g$. Moreover, the coalition formation game $g$ rejects all but finitely many core allocations of $v$ other than $\omega$. In case $v$ is strictly convex, the TU- $\alpha$-core, the TU- $\beta$-core and the set of all TU-strong equilibria of $g$ all become singletons. That is, $\omega$ now turns out to be the unique core allocation that survives in the strategic framework of $g$, while all other core allocations are killed by that same strategic environment. As, for each core allocation of $v$, there is a strategic environment that respects it, while rejecting the others, we say that every member of the core is as respectful as any other.

The family of coalition formation games, which we utilize in strategically separating different core allocations from each other, is then modified to now separate core outcomes from noncore outcomes by employing the TU-strong equilibrium notion in the arising strategic form games.

The rest of the paper is organized in a simple fashion. Section 2 introduces and defines the basic notions of the paper. In Sect. 3, we state and prove our results, while Sect. 4 closes the paper.

\section{Preliminaries}

We let $N$ stand for a nonempty finite set (of players) throughout the paper. A strategic form game is an ordered triple $g=(N, X, u)$, where $X_{i}$ is a nonempty (strategy) set and $u_{i}: X \rightarrow \mathbb{R}$ a (utility) function for each $i \in N$ with $X=\prod_{i \epsilon N} X_{i}$ and $u=\left(u_{i}\right)_{i \in N}$. We also set $X_{S}=\prod_{i \epsilon S} X_{i}$ for each $S \in 2^{N} \backslash\{\emptyset\}$.

A transferable utility (TU) game is a function $v: 2^{N} \rightarrow \mathbb{R}$ with $v(\emptyset)=0$. We say that a TU game $v$ is convex if and only if, for all $S, T \in 2^{N}$, one has $v(S)+v(T) \leq v(S \cup T)+v(S \cap T)$. Given a TU game $v$, we refer to $y \in \mathbb{R}^{N}$ as a core allocation of $v$ if and only if $\sum_{i \in N} y_{i}=v(N)$ and $\sum_{i \in S} y_{i} \geq v(S)$ for all $S \in 2^{N}$. We denote the set of all core allocations of a TU game $v$ by core $v$.

Let $g=(N, X, u)$ be a strategic form game such that, for each $S \in 2^{N} \backslash\{\emptyset\}, v_{g}^{\alpha}(S)=$ $\operatorname{Max}_{x_{S} \in X_{S}} \operatorname{Min}_{x_{N \backslash S} \in X_{N \backslash S}} \sum_{i \in S} u_{i}\left(x_{S}, x_{N \backslash S}\right)$ exists. Setting $v_{g}^{\alpha}(\emptyset)=0$, we obtain a 
TU game $v_{g}^{\alpha}$, referred to as the TU game induced by $g$ via the maxmin operator. For each $S \in 2^{N} \backslash\{\emptyset\}, v_{g}^{\alpha}(S)$ represents the maximal total utility that coalition $S$ can secure for itself, no matter what the complementary coalition $M S$ does.

We now imagine a situation where any coalition $S \in 2^{N} \backslash\{\emptyset\}$ can somehow anticipate the joint action $x_{N \backslash S} \in X_{N \backslash S}$ of $N \backslash S$ and choose its own joint strategy $x_{S} \epsilon X_{S}$ in response to $x_{N \backslash S}$. Assume that $g=(N, X, u)$ is a strategic form game such that $v_{g}^{\beta}(S)=\operatorname{Min}_{x_{N \backslash S} \in X_{N \backslash S}} \operatorname{Max}_{x_{S} \in X_{S}} \sum_{i \in S} u_{i}\left(x_{S}, x_{N \backslash S}\right)$ exists for each $S \in 2^{N} \backslash\{\emptyset\}$. When we again set $v_{g}^{\beta}(\emptyset)=0, v_{g}^{\beta}$, will be referred to as the TU game induced by $g$ via the minmax operator.

To remind the reader of the definitions of the $\alpha$-core, $\beta$-core and strong equilibrium by Aumann (1961), let $g=(N, X, u)$ be a strategic form game, $x^{*} \in X$ and $S \in 2^{N} \backslash\{\emptyset\}$. We say that $S \alpha$-blocks $x^{*}$ if and only if there is some $x_{S} \in X_{S}$ such that, for any $x_{N \backslash S} \in X_{N \backslash S}$, one has $u_{i}\left(x_{S}, x_{N \backslash S}\right) \geq u_{i}\left(x^{*}\right)$ for all $i \in S$, where at least one of the inequalities is strict. On the other hand, $S$ is said to $\beta$-block $x^{*}$ if and only if, for any $x_{N \backslash S} \in X_{N \backslash S}$, there is some $x_{S} \in X_{S}$ such that $u_{i}\left(x_{S}, x_{N \backslash S}\right) \geq u_{i}\left(x^{*}\right)$ for all $i \epsilon S$, where again at least one of the inequalities is strict. Moreover, $x^{*}$ is said to belong to the $\alpha$-core $C_{\alpha}(g)$ of $g$ if and only if there is no $S \in 2^{N} \backslash\{\emptyset\}$, which $\alpha$-blocks $x^{*}$. The definition of the $\beta$-core $C_{\beta}(g)$ of $g$ is obtained from the above by simply replacing $\alpha$ by $\beta$. Finally $x^{*}$ is said to be a strong equilibrium of $g$ if and only if, for any $S \in 2^{N} \backslash\{\emptyset\}$ and $x_{S} \in X_{S}$, one has $u_{i}\left(x^{*}\right) \geq u_{i}\left(x_{S}, x_{N \backslash S}^{*}\right)$ for all $i \in S$.

Aumann (1961) introduced the notions of $\alpha-\beta$-core and strong equilibrium as natural strategic counterparts of the core. In a strategic environment it becomes necessary to specify whether a coalition, which tries to improve upon a given outcome, chooses its joint action as a first, second or simultaneous mover against the complementary coalition. It is this distinction that leads to the three different strategic counterparts of the core.

Strategic form games have so far been almost always accompanied by nontransferability of utility. Aumann's (1961) strategic-cooperative notions are also based on this assumption. A strategic structure, however, does not conceptually exclude the possibility of utility being transferable. Moreover, the maxmin and minmax operators employed in associating a TU game with a given strategic form game $g$ are based on the assumption that utility is transferable in $g$. We now formalize the notions of the $\alpha-, \beta$-core and strong equilibrium in a strategic form game under transferable utility. Note that the comparison of two joint strategies $x$ and $x^{\prime}$ in $g$ by a coalition $S$ will get reduced to comparing the magnitudes $\sum_{i \epsilon S} u_{i}(x)$ and $\sum_{i \epsilon S} u_{i}\left(x^{\prime}\right)$, when utility is transferable.

Definition 1 Let $g=(N, X, u)$ be a strategic form game, $x^{*} \in X$ and $S \in 2^{N} \backslash\{\emptyset\}$. We say that $S$ TU- $\alpha$ blocks $x^{*}$ if and only if there is some $x_{S} \in X_{S}$ such that $\sum_{i \in S} u_{i}\left(x_{S}, x_{N \backslash S}\right)>\sum_{i \in S} u_{i}\left(x^{*}\right)$ for any $x_{N \backslash S} \in X_{N \backslash S}$. Similarly, $S$ will be said to TU- $\beta$ block $x^{*}$ if and only if, for each $x_{N \backslash S} \in X_{N \backslash S}$, there is some $x_{S} \in X_{S}$ with $\sum_{i \in S} u_{i}\left(x_{S}, x_{N \backslash S}\right)>\sum_{i \in S} u_{i}\left(x^{*}\right)$. We say that $x^{*} \in X$ belongs to the TU$\alpha$-core $C_{\alpha}^{1}(g)$ [resp., the TU- $\beta$-core $C_{\beta}^{1}(g)$ ] of $g$ if and only if there is no coalition $S \in 2^{N} \backslash\{\emptyset\}$, which TU- $\alpha$ [resp., TU- $\beta$ ] blocks $x^{*}$. Finally $x^{*}$ is said to be a TU 
strong equilibrium of $g$ if and only if, for any $S \in 2^{N} \backslash\{\emptyset\}$ and $x_{S} \in X_{S}$, one has $\sum_{i \in S} u_{i}\left(x^{*}\right) \geq \sum_{i \in S} u_{i}\left(x_{S}, x_{N \backslash S}^{*}\right)$.

It is now a straightforward to see that $C_{\alpha}^{1}(g)$ and $C_{\beta}^{1}(g)$ are the strategic counterparts of the cores of $v_{g}^{\alpha}$ and $v_{g}^{\beta}$, resp., as is formally reflected by the following proposition.

Proposition Let $g=(N, X, u)$ be a strategic form game.

(i) If $g$ induces a well-defined TU game $v_{g}^{\alpha}$ via the maxmin operator and $x^{*} \in C_{\alpha}^{1}(g)$, then $\left(u_{i}\left(x^{*}\right)\right)_{i \in N} \in$ core $v_{g}^{\alpha}$.

(ii) If $g$ induces a well-defined TU game $v_{g}^{\beta}$ via the minmax operator and $x^{*} \in C_{\beta}^{1}(g)$, then $\left(u_{i}\left(x^{*}\right)\right)_{i \in N} \in$ core $v_{g}^{\beta}$.

If a core allocation $\omega$ of $v_{g}^{\alpha}$ is induced by a joint strategy in the TU- $\alpha$-core $C_{\alpha}^{1}(g)$ of $g$, then this means that $\omega$ survives in the strategic environment of $g$. On the other hand, those core allocations of $v_{g}^{\alpha}$, which are not induced by any member of $C_{\alpha}^{1}(g)$ are eliminated by that same strategic environment of $g$. Thus, $g$ will be strategically separating some core allocations of $v_{g}^{\alpha}$ from others. The situation remains the same when we replace $\alpha$ with $\beta$.

For any given TU game $v$, there are an infinite variety of strategic form games $g$ with $v=v_{g}^{\alpha}$ [resp., $v=v_{g}^{\beta}$ ]. If a TU game $v$ is convex and $\omega, \omega^{\prime} \in$ core $v$ with $\omega$ $\neq \omega^{\prime}$, then a natural question that arises is whether there is a strategic game $g$ with $v=v_{g}^{\alpha}$ [resp., $v=v_{g}^{\beta}$ ] such that $g$ separates $\omega$ and $\omega^{\prime}$ in the sense that $\omega$ is induced by a joint strategy in $C_{\alpha}^{1}(g)$ [resp., $C_{\beta}^{1}(g)$ ], while $\omega^{\prime}$ is not. We will now construct a canonical family of strategic form games to strategically separate core outcomes from each other.

\section{An illustrative example}

The central idea underlying our approach is the introduction of transferable utility to strategic form games. Be it the maxmin or the minmax operator that one employs to obtain a TU game from a given strategic form game, the strategic aspects of the game get lost, although some of them may yet carry some relevant information concerning the core.

It might be best to consider an example to illustrate this phenomenon before we deal with general results. Now consider the following two strategic form games $G_{1}$ and $G_{2}$, where $G_{1}$ is the classical Prisoners' Dilemma, while $G_{2}$ is obtained from $G_{1}$ by modifying the payoffs in a particular way. ${ }^{1}$

\footnotetext{
${ }^{1}$ Player 1 is the row player as usual, where the first components in each cell refer to his payoff at that outcome. Player 2 is the column player whose payoffs are represented by the second component in each cell.
} 


\begin{tabular}{lll}
\hline & $G_{1}$ & \\
\cline { 2 - 3 } & $C$ & $D$ \\
\hline$C$ & $(1,1)$ & $(3,0)$ \\
$D$ & $(0,3)$ & $(2,2)$ \\
\hline
\end{tabular}

\begin{tabular}{lll}
\hline & $G_{2}$ & \\
\cline { 2 - 3 } & $C$ & $D$ \\
\hline$C$ & $(1,1)$ & $(3,0)$ \\
$D$ & $(0,3)$ & $(1,3)$ \\
\hline
\end{tabular}

First note that both $G_{1}$ and $G_{2}$ induce the same TU game $v$ with $v(\{1\})=v(\{2\})=$ 1 and $v(\{1,2\})=4$ under both the maxmin and the minmax operators. The core of $v$ is given by core $v=\left\{\left(x_{1}, x_{2}\right) \in \mathbb{R}^{2}: x_{1}, x_{2} \geq 1, x_{1}+x_{2}=4\right\}$, which is simply the line segment joining $(1,3)$ and $(3,1)$ in $\mathbb{R}^{2}$ with midpoint $(2,2)$.

In either of the two strategic form games, the achievable payoff pair are those at the joint pure strategies, when utility is not transferable. In particular, the only core allocation achievable in $G_{1}$ without any transfers is $(2,2)$, the midpoint of core $v$. Let us now examine what happens when we keep the strategic structure, but allow agents to make transfers to each other. Be it the TU- $\alpha$-core, the TU- $\beta$-core or the TU strong equilibrium notion that we employ, the only equilibrium reached in $G_{1}$ is easily seen to be $(D, D)$, yielding the payoff allocation $(2,2)$. The simple reason is that, by making a transfer, none of the players can induce a behavior on the part of the other player that would change the equilibrium outcome so as to yield a net benefit for the transferring player.

The Shapley value, the nucleolus and the core-center also allocate $(2,2)$ to the players in the induced TU game. As $G_{1}$ is symmetric and $(2,2)$ is an achievable joint payoff in $G_{1}$, this allocation only seems to be "natural" and "fair".

Each of the above three core selections, however, continues to allocate $(2,2)$ "under $G_{2}$ " as well, since they only care about the TU game $v$ induced by $G_{2}$, so that the change in the strategic background goes unnoticed by them. On the other hand, when we allow the players to make transfers in the strategic framework of $G_{2}$, the TU- $\alpha$ and the TU- $\beta$-cores as well as the set of TU-strong equilibria consists of $(D, D)$ only, yielding the allocation $(1,3)$, which is the core allocation of $v$ that favors player 2 most. The simple reason why the second player is "favored by the core under $G_{2}$ " is that the strategic structure of $G_{2}$ favors player 2 .

The reader now can easily see that something similar would happen if we change the payoff vector at $(D, D)$ to any $\left(\bar{x}_{1}, \bar{x}_{2}\right) \in \mathbb{R}^{2}$ with $\bar{x}_{1}, \bar{x}_{2} \geq 1$ and $\bar{x}_{1}+\bar{x}_{2}=4$. That is, the TU game induced would be identical with that induced by the Prisoners' Dilemma; the change in the strategic structure would go unnoticed by the three core selections considered; and the unique payoff allocation resulting from the TU- $\alpha$-core, the TU- $\beta$-core and the TU-strong equilibrium notion would be $\left(\bar{x}_{1}, \bar{x}_{2}\right)$. 
In other words, for each core allocation of the TU game $v$, we have a corresponding strategic environment, which allows only that core allocation to "survive", while all other core allocations of $v$ are "ruled out".

\section{Results}

Let $v$ be a convex TU game and $\omega \in$ core $v$. For each $i \in N$, set $X_{i}=\left\{S \in 2^{N} \mid i \in S\right\}$. For any $x \in X=\prod_{i \epsilon N} X_{i}$, we define a coalitional partition $\mathfrak{B}(x)$ of $N$ as follows: For any $T \in 2^{N}$ with $|T|>1$, we let $T \in \mathfrak{B}(x)$ if and only if $x_{i}=T$ for any $i \epsilon T$. For any $\mathrm{j} \in N$, we let $\{j\} \in \mathfrak{B}(x)$ if and only if either $x_{j}=\{j\}$ or $x_{k} \neq x_{j}$ for some $k \in x_{j}$. We say that $g=(N, X, u)$ is a canonical strategic form game for $(v, \omega)$ if and only if $u$ satisfies conditions (a) and (b) below:

Condition (a) If $x \in X$ is such that $x_{i}=N$ for all $i \in N$, then $u_{i}(x)=\omega_{i}$ for each $i$ $\epsilon N$.

Condition (b) If $x \in X$ is such that $x_{i} \neq N$ for some $i \in N$, then $u_{j}(x) \geq v(\{j\})$ for all $\mathrm{j} \epsilon N$, and $\sum_{j \in T} u_{j}(x)=v(T)$ for any $T \in \mathfrak{B}(x) .^{2}$

The existence of a canonical strategic form game for any $(v, \omega)$ as above follows from the convexity of $v$.

Theorem 1 Let $v$ be a convex TU game, $\omega \in$ core $v$ and $g=(N, X, u)$ a canonical strategic form game for $(v, \omega)$. Also let $\bar{x} \in X$ be such that $\bar{x}_{i}=N$ for all $i \in N$. Now

(a) $v_{g}^{\alpha}=v_{g}^{\beta}=v$.

(b) $\bar{x} \in C_{\alpha}^{1}(g) \cap C_{\beta}^{1}(g)$ and $\left(u_{i}(\bar{x})\right)_{i \in N}=\omega$.

Proof (a) First note that $v_{g}^{\alpha}(N)=v_{g}^{\beta}(N)=\operatorname{Max}_{x \in X} \sum_{i \in N} u_{i}(x)$ by definition of $v_{g}^{\alpha}$ and $v_{g}^{\beta}$. For any $x \in X \backslash\{\bar{x}\}$, one has $\mathfrak{B}(x) \neq\{N\}$, and thus $\sum_{T \in \mathfrak{B}(x)} v(T) \leq v(N)$ by convexity of $v$. On the other hand, $\sum_{i \in N} u_{i}(x)=\sum_{i \in N} \omega_{i}=v(N)$ by definition of $g$ and since $\omega \in$ core $v$. So, $\operatorname{Max}_{x \in X} \sum_{i \in N} u_{i}(x)=\sum_{i \in N} u_{i}(\bar{x})$, implying that $v_{g}^{\alpha}(N)=v_{g}^{\beta}(N)=v(N)$.

Now take any $S \in 2^{N} \backslash\{\varnothing, N\}$. Let $\tilde{x} \in X$ be such that $\tilde{x}_{i}=S$ for all $i \in S$ and $\tilde{x}_{j}=N \backslash S$ for all $\mathrm{j} \in N \backslash S$. Now $\sum_{i \in S} u_{i}\left(\tilde{x}_{S}, x_{N \backslash S}\right)=v(S)$ for all $x_{N \backslash S} \in X_{N \backslash S}$ since $\mathfrak{B}\left(\tilde{x}_{S}, x_{N \backslash S}\right)=\left\{S, T_{1}, \ldots, T_{l}\right\}$ for some partition $\left\{T_{1}, \ldots, T_{l}\right\}$ of $N \backslash S$. Thus, we have both $v_{g}^{\alpha}(S) \geq v(S)$ and $v_{g}^{\beta}(S) \geq v(S)$. On the other hand, for any $x_{S} \in X_{S}$, we have $\mathfrak{B}\left(x_{S}, \tilde{x}_{N \backslash S}\right)=\left\{N \backslash S, T_{1}, \ldots, T_{k}\right\}$ for some partition $\left\{T_{1}, \ldots, T_{k}\right\}$ of $S$. By definition of $g$, it follows that $\sum_{i \in S} u_{i}\left(x_{S}, \tilde{x}_{N \backslash S}\right)=\sum_{l=1}^{k} v\left(T_{l}\right) \leq v(S)$ by convexity of $v$. So, $v_{g}^{\beta}(S) \leq v(S)$. As $v_{g}^{\alpha}(S) \leq v_{g}^{\beta}(S)$, we also conclude that $v_{g}^{\alpha}(S) \leq v(S)$. Therefore, we have $v_{g}^{\alpha}(S)=v(S)=v_{g}^{\beta}(S)$ for all $S \in 2^{N} \backslash\{\emptyset\}$.

(b) Suppose that $\bar{x} \notin C_{\beta}^{1}(g)$. Now there is some $S \in 2^{N} \backslash\{\emptyset\}$, which TU- $\beta$ blocks $\bar{x}$. In particular, there is some $x_{S} \in X_{S}$ such that $\sum_{i \in S} u_{i}\left(x_{S}, \tilde{x}_{N \backslash S}\right)>\sum_{i \in S} u_{i}(\bar{x})=$ $\sum_{i \in S} \omega_{i} \geq v(S)$, since $\omega \in$ core $v$. Recall that $\mathfrak{B}\left(x_{S}, \tilde{x}_{N \backslash S}\right)=\left\{N \backslash S, T_{1}, \cdots, T_{k}\right\}$ for

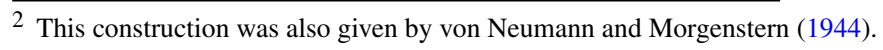


some partition $\left\{T_{1}, \ldots, T_{k}\right\}$ of $S$. We thus also have $\sum_{i \in S} u_{i}\left(x_{S}, \tilde{x}_{N \backslash S}\right)=\sum_{l=1}^{k} v\left(T_{l}\right)$ by definition of $g$, while $\sum_{l=1}^{k} v\left(T_{l}\right) \leq v(S)$ by convexity of $v$, yielding a contradiction. Thus, $\bar{x} \in C_{\beta}^{1}(g)$. Since clearly $C_{\beta}^{1}(g) \subset C_{\alpha}^{1}(g)$, we have $\bar{x} \in C_{\alpha}^{1}(g)$ as well. Moreover $\left(u_{i}(\bar{x})\right)_{i \in N}=\omega$ by condition (a).

Whatever core allocation $\omega$ of a convex TU game $v$ we start with, we now have a strategic form coalition formation game $g$ in which $\omega$ survives. As $g$ is finite, the TU- $\alpha$ and TU- $\beta$ cores of $g$ are also finite sets. In case $v$ has nonsingleton core, this simply means that infinitely many (in fact, all but finitely many) core allocations are rejected by the strategic environment of $g$. The next natural question this observation gives rise to is whether one can find a strategic environment for each core allocation $\omega$ such that $\omega$ survives, but all other core allocations are killed. Our canonical family of strategic form games provides such an environment for strictly convex TU games.

Definition 2 A TU game $v: 2^{N} \rightarrow \mathbb{R}$ is said to be strictly convex if and only if, for any $S, T \in 2^{N}$ with $S \not \subset T$ and $T \not \subset S$, one has $v(S)+v(T)<v(S \cup T)+v(S \cap T)$.

Corollary Let $v$ be a strictly convex $T U$ game and $\omega \in$ core $v$. If $g=(N, X, u)$ is a canonical strategic form game for $(v, \omega)$, then $C_{\alpha}^{1}(g)=C_{\beta}^{1}(g)=\{\bar{x}\}$ for some $\bar{x} \in X$ with $\omega=\left(u_{i}(\bar{x})\right)_{i \in N}$.

Proof Suppose that $x, y \in C_{\alpha}^{1}(g)$ with $x \neq y$ for some canonical game $g=(N, X, u)$ for $(v, \omega)$. By definition of $g$, we have $\mathfrak{B}(x) \neq\{N\}$ or $\mathfrak{B}(y) \neq\{N\}$. Assume without loss of generality that $\mathfrak{B}(x)=\left\{T_{1}, \ldots, T_{l}\right\}$ with $l>1$. Again by construction of $g$, we have $\sum_{j \in T_{k}} u_{j}(x)=v\left(T_{k}\right)$ for each $k \epsilon\{1, \ldots, l\}$. Thus $\sum_{j \in N} u_{j}(x)=\sum_{k=1}^{l} v\left(T_{k}\right)<v(N)$, where the last strict inequality follows from the strict convexity of $v$. Then, however, $N$ TU- $\alpha$ blocks $x$ by playing $\bar{x} \in X$ with $\bar{x}_{i}=N$ for each $i \epsilon N$, in contradiction with $x \in C_{\alpha}^{1}(g)$. As we already know that $\bar{x} \in C_{\alpha}^{1}(g)$ we conclude that $C_{\alpha}^{1}(g)=\{\bar{x}\}$ with $\left(u_{i}(\bar{x})\right)_{i \in N}=\omega$. Finally since $\bar{x} \in C_{\beta}^{1}(g) \subset C_{\alpha}^{1}(g)$, it also follows that $C_{\beta}^{1}(g)=\{\bar{x}\}$.

As the process of inducing a TU game $v_{g}^{\alpha}$ from a strategic form game $g$ via the maxmin operator follows the same rationale as the $\alpha$-core, we have associated the TU- $\alpha$ core of $g$ with the core of $v_{g}^{\alpha}$, where a similar reasoning naturally applies to the $\beta$-case as well. In the strategic framework provided by our canonical family of strategic form games, however, the distinction between the $\alpha$ - and $\beta$-approaches disappeared. The simultaneous-move counterpart of the core in a strategic environment is the notion of strong Nash equilibrium, whose transferable-utility version is defined as follows:

Definition 3 Let $g=(N, X, u)$ be a strategic form game and $\bar{x} \in X$. We say that $\bar{x}$ is a TU-strong equilibrium of $g$ if and only if, for any $S \in 2^{N} \backslash\{\emptyset\}$ and $x_{S} \in X_{S}$, one has $\sum_{i \in S} u_{i}(\bar{x}) \geq \sum_{i \in S} u_{i}\left(x_{S}, \bar{x}_{N \backslash S}\right)$.

Theorem 2 Let $v$ be a convex $T U$ game, $\omega \in$ core $v$ and $g=(N, X, u)$ a canonical strategic form game for $(v, \omega)$. Also let $\bar{x} \in X$ be such that $\bar{x}_{i}=N$ for all $i \in N$. Now $\bar{x}$ is a TU-strong equilibrium of $g$ with $\left(u_{i}(\bar{x})\right)_{i \in N}=\omega$. Moreover, if $v$ is strictly convex, then $\bar{x}$ is the unique TU-strong equilibrium of $g$. 
Proof Suppose that there are some $S \in 2^{N} \backslash\{\emptyset\}$ and $x_{S} \in X_{S}$ such that $\sum_{i \in S} u_{i}\left(x_{S}, \bar{x}_{N \backslash S}\right)>\sum_{i \in S} u_{i}(\bar{x})$. Then $\mathfrak{B}\left(x_{S}, \bar{x}_{N \backslash S}\right)=\left\{T_{1}, \ldots, T_{l}\right\} \cup$ $\{\{j\} \mid j \in N \backslash S\}$ for some partition $\left\{T_{1}, \ldots, T_{l}\right\}$ of $S$. Now, however, $\sum_{i \in S} u_{i}\left(x_{S}, \bar{x}_{N \backslash S}\right)=\sum_{k=1}^{l} v\left(T_{k}\right) \leq v(S)$ by definition of $g$ along with the convexity of $v$. On the other hand, $\sum_{i \in S} u_{i}(\bar{x})=\sum_{i \in S} \omega_{i} \geq v(S)$ since $\omega \in$ core $v$, yielding a contradiction. Thus, $\bar{x}$ is a TU-strong equilibrium of $g$.

Now assume that $v$ is strictly convex. Suppose that there is a TU-strong equilibrium $y \in X$ of $g$ with $y \neq \bar{x}$. Then, however, $\mathfrak{B}(y) \neq\{N\}$, i.e., $\mathfrak{B}(y)=\left\{T_{1}, \ldots, T_{l}\right\}$ with $l>1$. But now $\sum_{i \in N} u_{i}(y)=\sum_{k=1}^{l} v\left(T_{k}\right)<v(N)$ by strict convexity of $v$, contradicting that $y$ is a TU-strong equilibrium.

Whichever of the three natural strategic counterparts of the core we take, i.e., be it the TU- $\alpha$ core, the TU- $\beta$ core or the TU-strong equilibrium, our family of canonical games strategically separates different core allocations from each other.

We have so far been interested in strategically separating different core outcomes from each other. The canonical family of strategic form games associated with each pair $(v, \omega)$, where $v$ is a convex game and $\omega \in \operatorname{core} v$, however, paves also the ground for strategically separating core outcomes from noncore outcomes.

Given a convex TU game $v$, instead of specifying a particular core allocation of $v$, we now construct a strategic form game the set of whose TU-strong equilibria turns out to yield all core allocations of $v$.

Let $v$ be a convex TU game. For each $i \in N$, set $X_{i}=$ $\left\{(S, \omega) \in 2^{N} \times \mathbb{R}^{S} \mid i \in S\right.$ and $\left.\sum_{i \in S} \omega_{i}=v(S)\right\}$. Given any $x \in X=\prod_{i \in N} X_{i}$, we write $x_{i}=\left(S^{i}, \omega^{i}\right)$ for each $i \in N$. We define, for any $i \in N$,

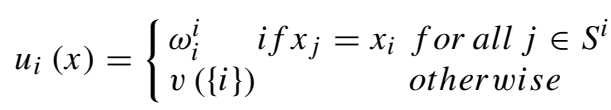

at each $x \in X$. We refer to $(N, X, u)$ as the strategic form game induced by $v$ and denote it by $g_{v}$.

The game $g_{v}$ induced by a convex TU game $v$ can also be described by the following scenario. Once every player $i \in N$ picks a strategy $x_{i} \in X_{i}$, the joint strategy $x$ leads to a coalition structure and a feasible distribution of the worth of any coalition in this partition among its members. Formally, denoting this "outcome function" by $h$, we have, for each $x \in X, h(x)=\left\{\left(T_{1}, \omega^{T_{1}}\right), \ldots,\left(T_{l}, \omega^{T_{l}}\right)\right\}$, where $\mathfrak{B}(x)=\left\{T_{1}, \ldots, T_{l}\right\}$ is a partition of $N$ and $\omega^{T_{k}} \in \mathbb{R}^{T_{k}}$ with $\sum_{i \in T_{k}} \omega_{i}^{T_{k}}=v\left(T_{k}\right)$ for each $k \in\{1, \ldots, l\}$ defined as follows in a similar fashion to our canonical games. For any $T \in 2^{N}$ with $|T|>1$, we let $T \in \mathfrak{B}(x)$ if and only if $x_{i}=\left(T, \omega^{T}\right)$ for all $i \in T$. For any $j \in N$, we let $\{j\} \in \mathfrak{B}(x)$ if and only if $x_{j}=(\{j\}, v(\{j\}))$ or $x_{k} \neq x_{j}$ for some $\mathrm{k} \in S^{j}$. For nonsingleton coalitions $T \in \mathfrak{B}(x), \omega^{T}$ is the allocation agreed upon by the members of $T$ via their declarations. A singleton coalition $\{i\} \in \mathfrak{B}(x)$ receives $v(\{i\})$. The utility profile $u$ of $g_{v}$ above summarizes the outcome of this process.

We denote the set of all TU-strong equilibria of a strategic form game $g$ by $S E_{T U}(g)$.

Theorem 3 For any convex $T U$ game $v$, one has $u\left(S E_{T U}\left(g_{v}\right)\right)=$ core $v$. 
Proof Take any $\omega \in \operatorname{core} v$. Let $\bar{x} \in X$ be such that $\bar{x}_{i}=(N, \omega)$ for each $i$ $\epsilon N$. Take any $S \in 2^{N} \backslash\{\emptyset\}$ and $x_{S} \in X_{S}$ with $x_{S} \neq \bar{x}_{S}$. Now $\mathfrak{B}\left(x_{S}, \bar{x}_{N \backslash S}\right)=$ $\left\{T_{1}, \ldots, T_{l}\right\} \cup\{\{j\} \mid j \in N \backslash S\}$ for some partition $\left\{T_{1}, \ldots, T_{l}\right\}$ of $S$, implying that $\sum_{i \in S} u_{i}\left(x_{S}, \bar{x}_{N \backslash S}\right)=\sum_{k=1}^{l} v\left(T_{k}\right) \leq v(S)$ by convexity of $v$. As $\omega \in$ core $v$, we also have $v(S) \leq \sum_{i \in S} w_{i}=\sum_{i \in N} u_{i}(\bar{x})$ since clearly $u_{i}(\bar{x})=\omega_{i}$ by definition of $g_{v}$. Thus $\sum_{i \in S} u_{i}\left(x_{S}, \bar{x}_{N \backslash S}\right) \leq \sum_{i \in S} u_{i}(\bar{x})$. So $\bar{x} \in S E_{T U}\left(g_{v}\right)$ with $u_{i}(\bar{x})=\omega$.

Conversely, let $\bar{x} \in S E_{T U}\left(g_{v}\right)$. Now suppose that there is some $S \in 2^{N} \backslash\{\emptyset\}$ with $\sum_{i \in S} u_{i}(\bar{x})<v(S)$. Let $\omega \in \mathbb{R}^{S}$ be such that $\sum_{i \in S} \omega_{i}=v(S)$, and set $x_{i}=(S, \omega)$ for each $i \in S$. Now, however, $\sum_{i \in S} u_{i}\left(x_{S}, \bar{x}_{N \backslash S}\right)=\sum_{i \in S} \omega_{i}=v(S)>\sum_{i \in S} u_{i}(\bar{x})$, in contradiction with $\bar{x} \in S E_{T U}\left(g_{v}\right)$. Thus, $u(\bar{x}) \in$ core $v$, completing the proof.

\section{Conclusion}

The main result of the paper is that the core allocations of a convex TU game are indistinguishable regarding the existence of a strategic background respecting them. It is in that sense that every member of the core is as respectful as any other. The very fact that the family of canonical coalition formation games are such that they induce the same TU game via the maxmin and minmax operators along with the coincidence of their TU- $\alpha$, TU- $\beta$ cores and TU-strong equilibria leaves no space for ambiguity concerning what the strategic counterpart of the core to be employed is. The strict convexity of a TU game also guarantees strict separation between different core outcomes. Given a convex TU game $v$ along with a particular core allocation $\omega$, the utility profile of the associated coalition formation game not only depends upon $v$, but also upon $\omega$. We obtain the mechanism that "implements" the core from our canonical family of coalition formation games by making the payoff profile a function of the joint strategy instead of fixing it contingent upon a given core allocation.

One common feature that underlies all results obtained in this paper is that we do not confine transferability of utility to nonstrategic environments only, but we also assume it in strategic contexts. Concerning the problem that we deal with, this only seems natural, as the process of inducing a TU game from a strategic form game finds the transferability of strategic-context utilities acceptable. Moreover, cooperative interaction is also widely modeled under nontransferability of utility. Be it a noncooperative or cooperative environment, it seems rather strange to us that one confines himself to either full transferability or absolute nontransferability of utility. Dede and Koray (2016) introduces semitransferability of utility both in strategic and nonstrategic contexts and, in particular, also deals with the counterparts of the problems of this paper. The light that Dede and Koray (2016) shed on the present study is that the simplicity of our results here is mainly driven by the full transferability of utility.

Acknowledgements BIDEB-2211 Graduate Scholarship Program of the Scientific and Technological Research Council of Turkey (TUBITAK) and Foundation for Economic Design are gratefully acknowledged for financial support to Y.D. 


\section{References}

Aumann R (1961) The core of a cooperative game without side payments. Trans Am Math Soc 98:539-552

Dede Y, Koray S (2016) Strategic separation of core outcomes under semitransferability of utility. Personal communication, Bilkent University, Turkey

Kalai E, Postlewaite A, Roberts J (1979) A group incentive compatible mechanism yielding core allocations. J Econ Theory 20:13-22

Lagunoff RD (1994) A simple noncooperative core story. Games Econ Behav 7:54-61

Perez-Castrillo JD (1992) Cooperative outcomes through noncooperative games. Games Econ Behav 7:428-440

Perry M, Reny PJ (1994) A noncooperative view of coalition formation and the core. Econometrica 62:795-817

Selten R (1975) Reexamination of the perfectness concept for equilibrium points in the extensive games. Int J Game Theory 4:25-55

Serrano R (1995) A market to implement the core. J Econ Theory 67:285-294

Von Neumann J, Morgenstern O (1944) Theory of games and economic behavior. Princeton University Press, Princeton 